\title{
Peripheral primitive neuroectodermal tumors: A retrospective analysis of 89 cases and literature review
}

\author{
LIMING GAO $^{1}$, YINGYING ZHU ${ }^{1}$, XIAOHUA SHI $^{2}$, ZHIQIANG GAO $^{1}$ and XINGMING CHEN ${ }^{1}$ \\ Departments of ${ }^{1}$ Otolaryngology-Head and Neck Surgery, and ${ }^{2}$ Pathology, Peking Union Medical College Hospital, \\ Peking Union Medical College and Chinese Academy of Medical Sciences, Beijing 100730, P.R. China
}

Received May 21, 2019; Accepted August 30, 2019

DOI: $10.3892 / \mathrm{ol} .2019 .11011$

\begin{abstract}
Peripheral primitive neuroectodermal tumors (pPNETs) are rare, small cell carcinomas with a poor prognosis. The aim of the present study was to describe therapeutic approaches, outcomes and probable prognostic factors. The clinical features, treatments, and outcomes of 89 consecutive patients with pPNET treated at the Peking Union Medical College Hospital from 1999 to 2018 were retrospectively reviewed. A total of 43 males and 46 females were included in the study, with a median age of 25 years (range, 5-73 years). The predominantly affected regions were the abdomen and pelvis, followed by the thoracopulmonary region. The mean primary tumor size was $12.6 \mathrm{~cm}$ (range, $1-30 \mathrm{~cm}$ ). A total of 16 patients (18\%) initially presented with metastasis. A total of 46 patients received combined therapy, 35 received mono-therapy, and 8 underwent only biopsy with no further treatment. The period of observation ranged from 1-232 months. The median overall survival (OS) time was 15 months [95\% confidence interval (CI), 9-21 months], with 3- and 5-year OS rates of 32 and 25\%, respectively. Large tumor size [adjusted hazard ratio $(\mathrm{aHR})=3.65$; 95\% CI, 2.07-6.42; $\mathrm{P}<0.001)$, metastasis at initial presentation $(\mathrm{aHR}=4.34,95 \% \mathrm{CI}, 2.23-8.42 ; \mathrm{P}<0.001)$, and combined modality treatment $(\mathrm{aHR}=0.16,95 \% \mathrm{CI}, 0.06-0.39 ; \mathrm{P}<0.001)$ were significantly associated with OS. The prognosis of pPNET is, overall, poor. Large tumor size and metastasis at initial presentation are associated with poorer outcomes. This highly malignant tumor requires an aggressive combination of radical resection, chemotherapy and radiotherapy, when indicated.
\end{abstract}

Correspondence to: $\mathrm{Dr}$ Xingming Chen, Department of Otolaryngology-Head and Neck Surgery, Peking Union Medical College Hospital, Peking Union Medical College and Chinese Academy of Medical Sciences, 1 Shuaifuyuan, Wangfujing, Beijing 100730, P.R. China

E-mail: xingming.chen@hotmail.com

Abbreviations: PNET, primitive neuroectodermal tumor; pPNET, peripheral PNET; HR, hazard ratio; OS, overall survival; PFS, progression-free survival

Key words: neuroectodermal tumors, primitive, peripheral, prognostic factors

\section{Introduction}

Primitive neuroectodermal tumors (PNET) are a rare, aggressive variant of small round cell carcinomas, primarily arising from neural crest cells. PNETs can be subdivided into central or peripheral (pPNET) types (1). pPNETs arise anywhere in the bone and soft tissues outside the central and sympathetic nervous system (2). They occur more frequently in adolescents and young adults with a slight male predominance, accounting for $4 \%$ of childhood and adolescent malignancies (3).

According to the World Health Organization, pPNETs, classic Ewing's sarcoma, and extraskeletal Ewing's sarcoma constitute the Ewing's sarcoma family of tumors. These tumors are defined by specific chromosomal abnormalities, primarily the $t(11,22)(\mathrm{q} 24,12)$ translocation $(4)$. Ewing's sarcoma and pPNET represent a spectrum of neuroectodermal differentiation, ranging from the least differentiated (Ewing's sarcoma) to the most differentiated (pPNET) (5). pPNETs may be distinguished from Ewing's sarcoma based on histological presentation and the expression of neural markers.

Histologically, pPNETs are comprised of small, round, darkly-stained cells possessing hyperchromatic nuclei, with a high mitotic rate (6). pPNETs may be confused with other small, round cell tumors, such as embryonal rhabdomyosarcoma, neuroblastoma or lymphoma. The presence of rosettes, particularly the typical Homer-Wright rosettes, indicates neural differentiation and aids in the diagnosis of pPNET (7). Immunohistochemically, 90-100\% of patients with pPNET are positive for CD99, the fusion gene product, which is a specific marker (4). Additional neural markers include neuron specific enolase, synaptophysin, chromogranin, neurofilament and S-100. In addition, electron microscopy of neurosecretory granules with microtubules and microfilaments are also supportive of a neural phenotype (7). The positive expression of at least 2 neural markers and/or Homer-Wright rosette formation is necessary for the diagnosis of pPNETs (8).

The actual incidence of pPNET is difficult to estimate due to its rarity. The majority of associated studies are case reports and clear diagnostic criteria have only recently been established (9). The estimated incidence of PNET is 2.9 per million per year (10). Due to its low incidence, there are presently no evidence-based guidelines for treatment. Despite the various treatments available, pPNETs maintain a poor prognosis. 
The present study comprised a retrospective analysis of all 89 patients with pPNET that were treated at Peking Union Medical College Hospital between January 1999 and December 2018, and comprehensively reviewed the literature to investigate clinical course, therapeutic approaches, and prognostic factors.

\section{Patients and methods}

Patients. A total of 89 patients with histologically diagnosed pPNET, hospitalized in Peking Union Medical College Hospital from January 1999 to December 2018, were retrospectively analyzed. The diagnoses of all cases were made by an experienced pathologist based on specimens obtained by surgery or biopsy, with the characteristic histology of a small, round cell tumor with formation of Homer-Wright rosettes, CD99 positivity, and markers of neuroepithelial differentiation. Cytogenetic evaluation for translocation $\mathrm{t}(11,22)(\mathrm{q} 24,12)$ was rarely performed $(1 / 89)$ in this cohort. The date of diagnosis recorded for each patient was the date of the earliest tumor biopsy from which the diagnosis was confirmed by local pathology review. Follow-up ended in March 2019. The study was approved by the Ethics Committee of Peking Union Medical College Hospital. Due to the retrospective nature of the study, the requirement for written informed consent was waived.

Clinical review. The primary site and size of the tumor, lymph node metastasis, distant metastasis, treatment details, and sex and age of the patient were evaluated by reviewing clinical records, imaging studies and surgical reports. Patients with insufficient data and those lost to follow-up were excluded from the review. Disease control intervals, survival and sites of relapse or metastasis were also assessed.

Statistical analysis. A database of all patients in the review was compiled and analyzed using SPSS v.21 software (IBM Corp.). Overall survival (OS) and progression-free survival (PFS) were calculated using the Kaplan-Meier method and compared using the log-rank test. OS was calculated from the date of diagnosis until the date of mortality or last follow-up. PFS was defined as the interval from diagnosis to disease progression, relapse, mortality, or last follow-up. A univariate Cox regression hazard model analysis assessed any predictors of outcome. Factors with significance $(\mathrm{P}<0.1)$ were taken into a stepwise multivariate Cox regression analysis, and the hazard ratios (HRs) and relevant $95 \%$ confidence intervals (CIs) were adjusted for age, sex, tumor size, tumor location, lymph nodes metastasis, distant metastasis and treatment strategies. $\mathrm{P}<0.05$ was considered to indicate a statistically significant difference.

Literature review. A comprehensive review of pPNET literature was undertaken using the PubMed (https://www.ncbi.nlm.nih. gov/pubmed) database for articles published in any year prior to December 2018, using the MeSH terms 'Neuroectodermal Tumors', 'Primitive' and 'Peripheral'. Additionally, the reference lists of associated articles were also screened to identify any potentially relevant studies. All relevant English language were retrieved and analyzed. Only studies with at least 10 patients were included and additional papers were identified from the citations in the retrieved articles. A total of 17 studies were selected for inclusion.
Table I. Demographic and clinical characteristics of the 89 patients with peripheral primary neuroectodermal tumors.

\begin{tabular}{lcr}
\hline Characteristics & No. of patients & $\%$ \\
\hline Age, years & & \\
Range (median) & $5-73(25)$ & \\
$<20$ & 32 & 36.0 \\
$\geq 20$ & 57 & 64.0 \\
Sex & & \\
Female & 46 & 51.7 \\
Male & 43 & 48.3 \\
Anatomical location & & \\
Thoracopulmonary & 25 & 28.1 \\
Abdomen/pelvic & 41 & 46.1 \\
Paravertebral & 7 & 7.9 \\
Head and neck & 7 & 7.9 \\
Extremities & 9 & 10.0
\end{tabular}

Size of primary tumor, $\mathrm{cm}$

$\begin{array}{lcc}\text { Range (mean) } & 1-30(12.6) & \\ \leq 10 & 44 & 49.4 \\ >10 & 45 & 50.6 \\ \text { Lymph node metastasis } & & \\ \text { No } & 72 & 80.9 \\ \text { Yes } & 17 & 19.1\end{array}$

Distant metastasis

No

$\begin{array}{ll}73 & 82.0\end{array}$

Yes

Site of metastasis

Lung

4

Bone

Liver

Adrenal gland

1.1

Central nervous system

1.1

Multiple site

6.7

Therapy

No

Mono-therapy

9.0

Combined

8

39.3

51.7

\section{Results}

Clinical presentation. From 1999 to 2018, 89 patients with pPNET were treated at Peking Union Medical Hospital. All patient characteristics are summarized in Table I. There were 43 males and 46 females, with a male: Female ratio of 0.93 . A wide range of ages were affected. The median age at diagnosis was 25 years (range, 5-73 years). The mean tumor size at diagnosis was $12.6 \mathrm{~cm}$ (range, 1-30 cm) and the abdominal and pelvic region (46\%) was the predominant primary tumor location, followed by the thoracopulmonary area $(28 \%)$. Lymph node and distant metastases occurred in 17 (19\%) and 16 patients (18\%), respectively. Bone and lung tissues were the most common sites of distant metastasis. 
Table II. Univariate and multivariate risk factors for mortality among patients with peripheral primary neuroectodermal tumors.

\begin{tabular}{|c|c|c|c|c|}
\hline \multirow[b]{2}{*}{ Characteristics } & \multicolumn{2}{|c|}{ Univariate analysis $(\mathrm{n}=89)$} & \multicolumn{2}{|c|}{ Multivariate analysis $(\mathrm{n}=89)$} \\
\hline & HR $(95 \%$ CI $)$ & P-value & aHR (95\% CI) & P-value \\
\hline \multicolumn{5}{|l|}{ Age, years } \\
\hline$<20$ & 1.00 & & - & - \\
\hline$\geq 20$ & $1.90(1.08-3.32)$ & 0.025 & - & - \\
\hline \multicolumn{5}{|l|}{ Sex } \\
\hline Female & 1.00 & - & - & - \\
\hline Male & $1.39(0.84-2.31)$ & 0.200 & - & - \\
\hline \multicolumn{5}{|l|}{ Tumor size, cm } \\
\hline$\leq 10$ & 1.00 & - & 1.00 & - \\
\hline$>10$ & $2.54(1.50-4.30)$ & 0.001 & $3.65(2.07-6.42)$ & $<0.001$ \\
\hline \multicolumn{5}{|l|}{ Location } \\
\hline Thoracopulmonary & $1.19(0.47-2.98)$ & 0.714 & - & - \\
\hline Abdomen/pelvis & $1.11(0.46-2.67)$ & 0.825 & - & - \\
\hline Paravertebral & $1.25(0.38-4.11)$ & 0.712 & - & - \\
\hline Head and neck & $0.29(0.06-1.45)$ & 0.133 & - & - \\
\hline Extremities & 1.00 & - & - & - \\
\hline \multicolumn{5}{|l|}{ Lymph nodes } \\
\hline No & 1.00 & - & - & - \\
\hline Yes & $1.37(0.75-2.50)$ & 0.314 & - & - \\
\hline \multicolumn{5}{|l|}{ Metastasis } \\
\hline No & 1.00 & - & 1.00 & - \\
\hline Yes & $3.98(2.16-7.32)$ & $<0.001$ & $4.34(2.23-8.42)$ & $<0.001$ \\
\hline \multicolumn{5}{|l|}{ Therapy } \\
\hline No & 1.00 & - & 1.00 & - \\
\hline Mono-therapy & $0.38(0.17-0.86)$ & 0.019 & $0.67(0.28-1.59)$ & 0.358 \\
\hline Combined & $0.12(0.05-0.29)$ & $<0.001$ & $0.16(0.06-0.39)$ & $<0.001$ \\
\hline
\end{tabular}

CI, confidence interval; HR, hazard ratio; aHR, adjusted hazard ratio.

Treatment. Various treatment strategies were adopted. Combined therapy was administered in 46 patients $(52 \%)$, 35 received mono-therapy and 8 received biopsy only, with no further treatment. A total of 52 patients received combination chemotherapy in their primary treatment plan. The most commonly used chemotherapy strategy was cincristine, actinomycine, cyclophosphamide and doxorubicin, with ifosfamide and etoposide. Chemotherapy was administered on a 21-day schedule with a planned total of 17 courses. Surgery/radiotherapy was adopted for local control. A total of 70 patients $(79 \%)$ received surgery, among which 45 achieved wide resection with negative surgical margins. A total of 38/70 patients also underwent lymph node dissection at the same time, and 13 were positive for lymph node metastases. An additional 3 patients who were diagnosed with lymph nodes metastases based on imaging studies received chemotherapy, biopsy only, and subtotal resection without lymph node dissection, respectively. A total of 19 patients (21\%) received radiotherapy, including 3 with lymph node metastases and 1 with prostate pPNET with lung metastasis. A total of 10/19 patients obtained negative margins, 6 patients underwent subtotal resection, 1 patient received chemoradiotherapy following biopsy, and the remaining 2 patients were treated with radiotherapy alone. The doses ranged from 15.4-60 Gy, delivered 5 times per week in a 1.8-2.0 Gy/fraction.

Survival and prognostic factors. The follow-up time ranged from 1-232 months with a mean of 30 months (median, 13 months). All mortalities were caused by the disease and no secondary tumors occurred. The median OS duration of the entire group was 15 months (95\% CI, 9-21 months) and the median PFS was 10 months (95\% CI, 7-13 months). The estimated 3-year OS rate was $32 \%$ and the 3 -year PFS was $23 \%$.

Patients with tumor size $\leq 10 \mathrm{~cm}$ exhibited an improved OS compared with those whose tumor was $>10 \mathrm{~cm}(\mathrm{P}<0.001)$. All patients with distant metastasis succumbed to the disease, corresponding to a 1-year survival rate of $25 \%$, compared with $62 \%$ for patients with localized disease. Treatment strategy was strongly associated with outcome. The median OS for patients with combined therapy was 38 months (95\% CI, 19-57 months), compared with 9 months for mono-therapy (95\% CI, 5-13 months), and 4 months for patients with no therapy (95\% CI, 1-7 months). Younger patients ( $<20$ years) 


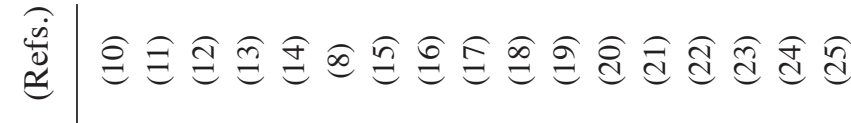

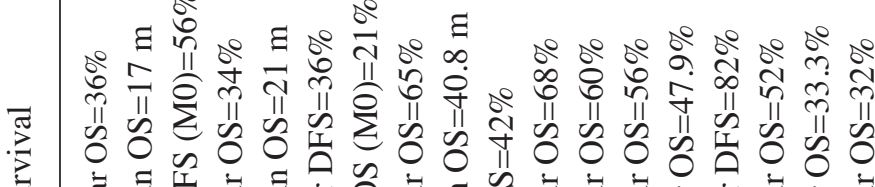

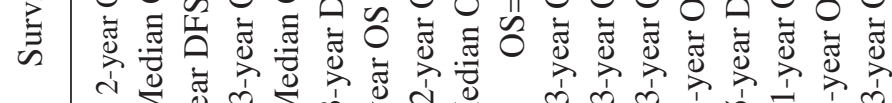

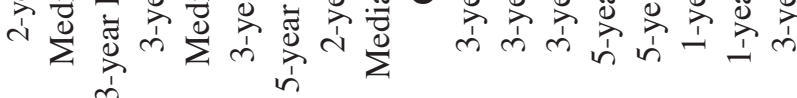

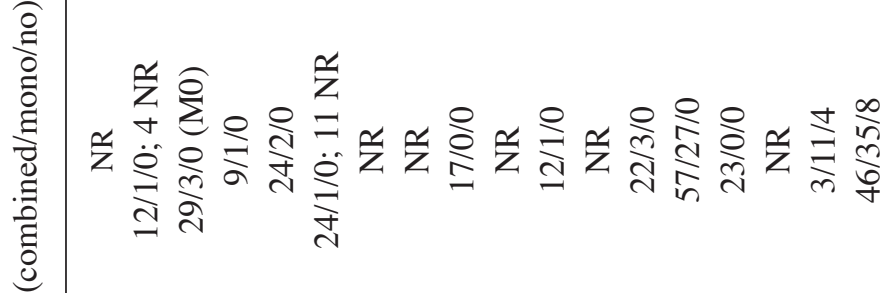

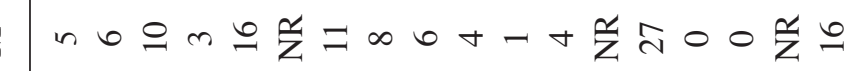

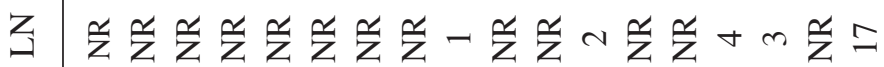

च

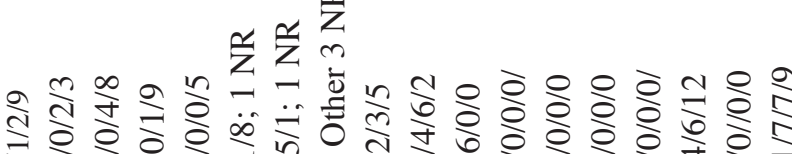

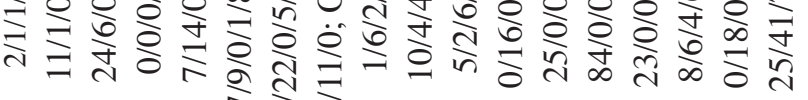

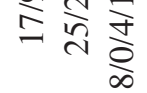

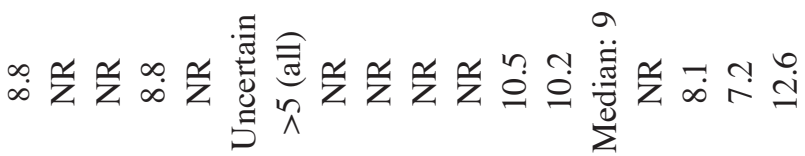

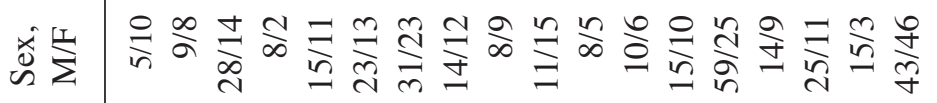

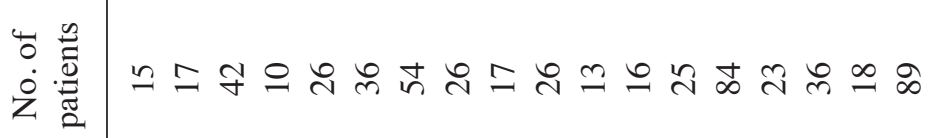


exhibited an improved prognosis ( $\mathrm{P}=0.025)$, but neither sex nor lymph node metastasis had any significant effect on survival.

The univariate and multivariate Cox regression analyses of the effect of possible prognostic factors on OS for all patients is summarized in Table II. Large tumor size [adjusted HR $(\mathrm{aHR})=3.65 ; 95 \%$ CI 2.07-6.42; $\mathrm{P}<0.001)$, distant metastasis at presentation $(\mathrm{aHR}=4.34 ; 95 \% \mathrm{CI}, 2.23-8.42 ; \mathrm{P}<0.001)$ and combined modality treatment $(\mathrm{aHR}=0.16 ; 95 \% \mathrm{CI}, 0.06-0.39$; $\mathrm{P}<0.001)$ were significantly associated with overall survival.

\section{Discussion}

Our review of the literature revealed 17 pPNET report series. The clinical features, treatments and outcomes of the reported patients, as well as the 89 in the present study, are summarized in Table III $(8,11-26)$.

According to the review conducted in the present study, pPNET affects all age groups, particularly adolescents and young adults, although certain studies restricted inclusion to young patients $(12,15,17,19)$. The median age ranged from 1 (19) to 36 (25) years. A male preponderance was described in 14 studies $(8,12-17,20-26)$. Nevertheless, there were a comparable 43 males and 46 females in the present study.

Diagnosis of pPNETs requires positive expression of at least 2 neural markers and/or Homer-Wright rosettes (8). In the patient cohort in the present study, all patients were positive for at least 2 neural markers, with a CD99 positivity of $93 \%$.

Thoracopulmonary tumors were more frequently observed in previously published studies, while the predominant location of pPNETs in the present study was the abdominal/pelvic region.

Peripheral PNETs are generally large, soft tissue masses with a reported mean diameter $>5 \mathrm{~cm}$, as confirmed by the reviewed studies that investigated primary tumor size $(11,14,16,21,22,25,26)$. Tumor bulk was the single most important prognostic factor, with $80 \%$ survival among patients with smaller primary tumors $(<100 \mathrm{ml})$, compared with only $40 \%$ survival among those with larger tumors (>100 ml) (27). The present study confirmed that the prognosis of patients with a tumor size $>10 \mathrm{~cm}$ was significantly poorer compared with those with a tumor size $<10 \mathrm{~cm}(\mathrm{aHR}=3.65 ; 95 \% \mathrm{CI}, 2.07-6.42$; $\mathrm{P}<0.001)$. The 3 -year OS rates for the two groups were 45 and $20 \%$, respectively.

A substantial proportion of patients presented with distant metastasis at diagnosis $(11,12,14,15,17,18,23)$. In the present study, all patients $(n=16 ; 18 \%)$ with metastasis succumbed to the disease during the follow-up period, corresponding to a 1-year survival of $25 \%$, compared with $62 \%$ for patients with localized disease. In the analysis of the present study, the location of metastasis at diagnosis was primarily the lungs and bones, which is consistent with previous studies $(13,14,17,25)$.

The rarity of pPNET makes it difficult to ascertain optimal management for these patients. However, improved outcomes are associated with more aggressive, combined modality treatments. In the present study, the median OS for patients with combined therapy was 38 months (95\% CI, 19-57 months) compared with only 9 months (95\% CI, 5-13 months) for mono-therapy and 4 months (95\% CI, 1-7 months) for patients with no therapy. Notably, the multivariate Cox regression analysis indicated that the outcome of patients with single modality treatment was not statistically different compared with that of patients receiving no treatment, indicating that single modality treatment is insufficient for the control of aggressive tumors like pPNET.

Surgical tumor resection with negative margins is an important priority for localized control and has been demonstrated to result in improved OS (2). Further, additional postoperative radiotherapy may control local disease for incomplete excisions and close tumor margins (28). However, the role of radiotherapy alone as a primary local treatment remains controversial. Even if the disease is controlled by complete resection, the metastatic spread may have already occurred. Aggressive treatment strategies, like combining systemic chemotherapy with surgery/radiotherapy, offer the best results. Since the 1980s, preliminary studies have suggested that the chemotherapeutic agents vincristine, ifosfamide, cyclophosphamide and doxorubicin are optimal for the treatment of metastatic Ewing's sarcoma. Likewise, identical chemotherapeutic strategies were performed on pPNETs to achieve improved survival rates (29). Generally, chemotherapy is administered as a neo-adjuvant or adjuvant therapy. Preoperative chemotherapy downstages the tumor in a majority of patients, thereby increasing the probability of achieving complete resection with microscopically negative margins, thereby decreasing the risk of intraoperative tumor cell dissemination $(20,30)$. Adjuvant chemotherapy, with or without radiotherapy, is recommended, regardless of surgical margins, following local treatment.

While previous studies have described similar results, the sample sizes of these previous studies were relatively small and the information was usually not sufficiently comprehensive. To the best of our knowledge, the present study included the largest sample size at present. Even though the present study was retrospective, increasing sample size may further decrease the statistical deviation, providing more reliable results.

In conclusion, pPNET is a highly aggressive tumor with a poor prognosis. Large tumor size and metastasis at primary presentation are associated with poorer outcomes. Combined treatment strategies, consisting of surgery, chemotherapy and radiation, remain controversial. The best combination of chemotherapy remains to be explored. Larger studies are required to confirm the results of the present study.

\section{Acknowledgements}

Not applicable.

\section{Funding}

No funding was received.

\section{Availability of data and materials}

The datasets used and/or during the present study are available from the corresponding author on reasonable request.

\section{Authors' contributions}

$\mathrm{XC}$ and $\mathrm{YZ}$ conceived the study. LG, XS and ZG acquired the data. LG, XC and YZ analyzed and interpreted the data. LG and YZ prepared the manuscript. All authors read and approved the final manuscript for publication. 


\section{Ethics approval and consent to participate}

The study was approved by the Ethics Committee of Peking Union Medical College Hospital, and written informed consent was waived, as patient data was retrospectively collected and anonymous.

\section{Patient consent for publication}

Due to the retrospective nature of the study, the requirement for written informed consent was waived.

\section{Competing interests}

The authors declare that they have no competing interests.

\section{References}

1. Virani MJ and Jain S: Primary intraspinal primitive neuroectodermal tumor (PNET): A rare occurrence. Neurol India 50 75-80, 2002.

2. Dehner LP: Peripheral and central primitive neuroectodermal tumors. A nosologic concept seeking a consensus. Arch Pathol Lab Med 110: 997-1005, 1986.

3. Coffin CM and Dehner LP: Peripheral neurogenic tumors of the soft tissues in children and adolescents: A clinicopathologic study of 139 cases. Pediatr Pathol 9: 387-407, 1989.

4. Ambros IM, Ambros PF, Strehl S, Kovar H, Gadner H and Salzer-Kuntschik M: MIC2 is a specific marker for Ewing's sarcoma and peripheral primitive neuroectodermal tumors. Evidence for a common histogenesis of Ewing's sarcoma and peripheral primitive neuroectodermal tumors from MIC2 expression and specific chromosome aberration. Cancer 67: 1886-1893, 1991.

5. Fletcher C: Peripheral neural tumors. In: Fletcher CDM, (eds). Diagnostic histopathology of tumors. Edinburgh: CHurchil Livingstone: 1239-1241, 1995.

6. Hachitanda Y, Tsuneyoshi M, Enjoji M, Nakagawara A and Ikeda K: Congenital primitive neuroectodermal tumor with epithelial and glial differentiation. An ultrastructural and immunohistochemical study. Arch Pathol Lab Med 114: 101-105, 1990

7. Votta TJ, Fantuzzo JJ and Boyd BC: Peripheral primitive neuroectodermal tumor associated with the anterior mandible: A case report and review of the literature. Oral Surg Oral Med Oral Pathol Oral Radiol Endod 100: 592-597, 2005.

8. Schmidt D, Herrmann C, Jurgens H and Harms D: Malignant peripheral neuroectodermal tumor and its necessary distinction from Ewing's sarcoma. A report from the Kiel Pediatric Tumor Registry. Cancer 68: 2251-2259, 1991.

9. Kalantari M, Deyhimi P and Kalantari P: Peripheral Primitive Neuroectodermal Tumor (pPNET) of the Parotid: Report of a rare case. Arch Iran Med 18: 858-860, 2015.

10. Ghosh A, Saha S, Pal S, Saha PV and Chattopadhyay S: Peripheral primitive neuroectodermal tumor of head-neck region: Our experience. Indian J Otolaryngol Head Neck Surg 61: 235-239, 2009.

11. Hashimoto H, Enjoji M, Nakajima T, Kiryu $H$ and Daimaru $Y$ Malignant neuroepithelioma (peripheral neuroblastoma). A clinicopathologic study of 15 cases. Am J Surg Pathol 7: 309-318, 1983.

12. Schmidt D, Harms D and Burdach S: Malignant peripheral neuroectodermal tumours of childhood and adolescence. Virchows Arch A Pathol Anat Histopathol 406: 351-365, 1985.

13. Jürgens H, Bier V, Harms D, Beck J, Brandeis W, Etspüler G, Gadner H, Schmidt D, Treuner J, Winkler K, et al: Malignant peripheral neuroectodermal tumors. A retrospective analysis of 42 patients. Cancer 61: 349-357, 1988.
14. Llombart-Bosch A, Terrier-Lacombe MJ, Peydro-Olaya A and Contesso G: Peripheral neuroectodermal sarcoma of soft tissue (peripheral neuroepithelioma): A pathologic study of ten cases with differential diagnosis regarding other small, round-cell sarcomas. Hum Pathol 20: 273-280, 1989.

15. Marina NM, Etcubanas E, Parham DM, Bowman LC and Green A: Peripheral primitive neuroectodermal tumor (peripheral neuroepithelioma) in children. A review of the St. Jude experience and controversies in diagnosis and management. Cancer 64: 1952-1960, 1989.

16. Kushner BH, Hajdu SI, Gulati SC, Erlandson RA, Exelby PR and Lieberman PH: Extracranial primitive neuroectodermal tumors. The memorial Sloan-Kettering cancer center experience. Cancer 67: 1825-1829, 1991.

17. Jones JE and McGill T: Peripheral primitive neuroectodermal tumors of the head and neck. Arch Otolaryngol Head Neck Surg 121: 1392-1395, 1995.

18. Ibarburen C, Haberman JJ and Zerhouni EA: Peripheral primitive neuroectodermal tumors. CT and MRI evaluation. Eur J Radiol 21: 225-232, 1996.

19. Kimber C, Michalski A, Spitz L and Pierro A: Primitive neuroectodermal tumours: Anatomic location, extent of surgery, and outcome. J Pediatr Surg 33: 39-41, 1998.

20. Zimmermann T, Blutters-Sawatzki R, Flechsenhar K and Padberg WM: Peripheral primitive neuroectodermal tumor: Challenge for multimodal treatment. World J Surg 25: 1367-1372, 2001.

21. Thyavihally YB, Tongaonkar HB, Gupta S, Kurkure PA, Amare P, Muckaden MA and Desai SB: Primitive neuroectodermal tumor of the kidney: A single institute series of 16 patients. Urology 71 : 292-296, 2008.

22. Demir A, Gunluoglu MZ, Dagoglu N, Turna A, Dizdar Y, Kaynak K, Dilege S, Mandel NM, Yilmazbayhan D, Dincer SI and Gurses A: Surgical treatment and prognosis of primitive neuroectodermal tumors of the thorax. J Thorac Oncol 4: 185-192, 2009.

23. Biswas B, Agarwala S, Shukla NK, Deo S, Sharma D, Thulkar S, Vishnubhatla S and Bakhshi S: Evaluation of outcome and prognostic factors in thoracic primitive neuroectodermal tumor: A study of 84 cases. Ann Thorac Surg 96: 2006-2014, 2013.

24. Sirivella S and Gielchinsky I: Treatment outcomes in 23 thoracic primitive neuroectodermal tumours: A retrospective study. Interact Cardiovasc Thorac Surg 17: 273-279, 2013.

25. Tan Y, Zhang H, Ma GL, Xiao EH and Wang XC: Peripheral primitive neuroectodermal tumor: Dynamic CT, MRI and clinicopathological characteristics-analysis of 36 cases and review of the literature. Oncotarget 5: 12968-12977, 2014.

26. Yi X, Liu W, Zhang Y, Xiao D, Yin H, Long X, Li L, Zai H, Chen M, Li W and Sun L: Radiological features of primitive neuroectodermal tumors in intra-abdominal and retroperitoneal regions: A series of 18 cases. PLoS One 12: e0173536, 2017.

27. Verrill MW, Judson IR, Harmer CL, Fisher C, Thomas JM and Wiltshaw E: Ewing's sarcoma and primitive neuroectodermal tumor in adults: Are they different from Ewing's sarcoma and primitive neuroectodermal tumor in children? J Clin Oncol 15: 2611-2621, 1997.

28. Rud NP, Reiman HM, Pritchard DJ, Frassica FJ and Smithson WA: Extraosseous Ewing's sarcoma. A study of 42 cases. Cancer 64: 1548-1553, 1989.

29. Wexler LH, DeLaney TF, Tsokos M, Avila N, Steinberg SM, Weaver-McClure L, Jacobson J, Jarosinski P, Hijazi YM, Balis FM and Horowitz ME: Ifosfamide and etoposide plus vincristine, doxorubicin, and cyclophosphamide for newly diagnosed Ewing's sarcoma family of tumors. Cancer 78: 901-911, 1996.

30. Shamberger RC, LaQuaglia MP, Gebhardt MC, Neff JR, Tarbell NJ, Marcus KC, Sailer SL, Womer RB, Miser JS, Dickman PS, et al: Ewing sarcoma/primitive neuroectodermal tumor of the chest wall: Impact of initial versus delayed resection on tumor margins, survival, and use of radiation therapy. Ann Surg 238: 563-568, 2003. 